\title{
Diamagnetism of quantum gases with singular potentials.
}

October 31,2018

\author{
Philippe Briet 11, Horia D. Cornean2, Baptiste Savoie ${ }^{3}$.
}

\begin{abstract}
We consider a gas of quasi-free quantum particles confined to a finite box, subjected to singular magnetic and electric fields. We prove in great generality that the finite volume grand-canonical pressure is analytic w.r.t. the chemical potential and the intensity of the external magnetic field. We also discuss the thermodynamic limit.
\end{abstract}

AMS 2000 Mathematics Subject Classification: 82B10, 82B2, 81V99.

Keywords: Diamagnetism, Schrödinger Operators, Gibbs ensembles.

\section{Introduction and the main result.}

The quest for rigorous results concerning the thermodynamic limit of the magnetic susceptibility of a gas of quasi-free quantum particles in the presence of a magnetic field started in 1975 with the work of Angelescu et. al. 1, 2]. Their method used in an essential way that the confining domain was a parallelepiped, the Hamiltonian was purely magnetic and the susceptibility was computed at zero magnetic field.

In a series of papers [13, 17, 8, 9, 15, 29] we gradually removed these constraints, and now we know how to prove the thermodynamic limit for generalized susceptibilities at arbitrarily large magnetic fields, and with smooth and periodic electric potentials. This achievement was possible due to a new idea, which led to the development of a systematic magnetic perturbation theory for Gibbs semigroups.

In this paper we examine the case in which both the magnetic field and the electric potential can have singularities, such that the magnetic and scalar singular perturbations are relatively bounded in the form sense with respect to the purely magnetic Schrödinger operator with constant magnetic field.

There is a huge amount of literature dedicated to spectral and statistical aspects of diamagnetism in large quantum systems. Some of the closely related papers to our work are [3, 4, 10, 12, 14, 21, 24.

Now let us introduce some notation and give the main theorem. Consider a magnetic vector potential $\mathbf{a}=\left(a_{1}, a_{2}, a_{3}\right)=\mathbf{a}_{c}+\mathbf{a}_{p}$ where $B \mathbf{a}_{c}:=\frac{B}{2} \mathbf{e} \times \mathbf{x}, \mathbf{e}=(0,0,1)$ is the usual symmetric gauge generated by a constant magnetic field $\mathbf{B}=B \mathbf{e}, B>0$ and $\mathbf{a}_{p}$ is $\mathbb{Z}^{3}$-periodic satisfying $\left|\mathbf{a}_{p}\right|^{2} \in \mathcal{K}_{\text {loc }}\left(\mathbb{R}^{3}\right)$. The notation $\mathcal{K}_{\text {loc }}$ denotes the usual Kato class [16, 30]. Relations between these assumptions on magnetic potentials and related choices of periodic magnetic fields are discussed in [24]. Assume that $V$ is also $\mathbb{Z}^{3}$-periodic such that $V \in \mathcal{K}_{l o c}\left(\mathbb{R}^{3}\right)$. Later on we will give a rigorous sense to the operator (here $\omega:=e B / c \in \mathbb{R}$ )

$$
H_{\infty}(\omega, V):=\frac{1}{2}(-i \nabla-\omega \mathbf{a})^{2}+V
$$

corresponding to the obvious quadratic form initially defined on $C_{0}^{\infty}\left(\mathbb{R}^{3}\right)$. If $\Lambda$ is a bounded open and simply connected subset of $\mathbb{R}^{3}$ we denote by $H_{\Lambda}(\omega, V)$ the operator obtained by restricting

\footnotetext{
${ }^{1}$ Université du sud Toulon-Var \& Centre de Physique Théorique, Luminy, Case 907 13288 Marseille CEDEX 9, France; e-mail: briet@univ-tln.fr

${ }^{2}$ Department of Mathematical Sciences, Aalborg University, Fredrik Bajers Vej 7G, 9220 Aalborg, Denmark; e-mail: cornean@math.aau.dk

${ }^{3}$ Centre de Physique Théorique, Luminy, Case 907, 13288 Marseille, CEDEX 9, France, and Department of Mathematical Sciences, Aalborg University, Fredrik Bajers Vej 7G, 9220 Aalborg, Denmark; e-mail: baptiste.savoie@gmail.com
} 
the above mentioned quadratic form to $C_{0}^{\infty}(\Lambda)$. We will see that $H_{\Lambda}(\omega, V)$ has purely discrete spectrum.

Let $\omega \in \mathbb{R}$ and $\beta:=\frac{1}{k_{B} T}>0$, where $T>0$ is the temperature and $k_{B}$ is the Boltzmann constant. Set $e_{0}=e_{0}(\omega)$ to be inf $\sigma\left(H_{\infty}(\omega, V)\right)$. Introduce the following complex domains

$$
D_{+1}\left(e_{0}\right):=\mathbb{C} \backslash\left(-\infty,-e^{\beta e_{0}}\right], \quad D_{-1}\left(e_{0}\right):=\mathbb{C} \backslash\left[e^{\beta e_{0}}, \infty\right)
$$

The grand canonical finite volume pressure is defined as [1, 3, 22,

$$
P_{\Lambda}(\beta, \omega, z, \epsilon)=\frac{\epsilon}{\beta|\Lambda|} \operatorname{Tr}_{L^{2}(\Lambda)}\left\{\ln \left(\mathbb{I}+\epsilon z e^{-\beta H_{\Lambda}(\omega, V)}\right)\right\}
$$

where $\epsilon=+1$ corresponds to the Fermi case and $\epsilon=-1$ corresponds to the Bose case. In (1.2) the activity $z \in D_{\epsilon}\left(e_{0}\right) \cap \mathbb{R}$. The operator $\ln \left(\mathbb{I}+\epsilon z e^{-\beta H_{\Lambda}(\omega, V)}\right)$ in the right hand side of (1.2) is defined via functional calculus. Due to some trace class estimates which we will prove later on (see (2.12) $)$, the pressure $P_{\Lambda}$ in (1.2) is well defined. Define the following complex domains

$$
\mathbf{D}_{\epsilon}:=\bigcap_{\omega \in \mathbb{R}} D_{\epsilon}\left(e_{0}(\omega)\right)=D_{\epsilon}\left(e_{0}(0)\right), \quad \epsilon= \pm 1
$$

Now we can formulate the main result of this paper:

Theorem 1.1. Let $\beta>0$.

(i). For each open set $K \subset \mathbb{C}$ with the property that $\bar{K}$ is compact and included in $\mathbf{D}_{\epsilon}$, there exists a complex neighborhood $\mathcal{N}$ of the real axis such that $\mathcal{N} \times K \ni(\omega, z) \mapsto P_{\Lambda}(\beta, \omega, z, \epsilon)$ is analytic.

(ii). Let $\omega \in \mathbb{R}$ and choose a compact set $K \subset D_{\epsilon}\left(e_{0}(\omega)\right)$. Then uniformly in $z \in K$

$$
P_{\infty}(\beta, \omega, z, \epsilon):=\lim _{\Lambda \rightarrow \mathbb{R}^{3}} P_{\Lambda}(\beta, \omega, z, \epsilon)
$$

exists and defines a smooth function of $\omega$.

In Theorem 1.1 (ii), we take the large volume limit in the sense defined in the section 3.4 below.

The rest of the paper contains the proof of this theorem. While (i) and the first part of (ii) will be proved quite in detail, we only outline the main ideas leading to the smoothness of $P_{\infty}$, all details will be given in 29].

\section{Technical preliminaries.}

Define the sesquilinear non-negative form on $C_{0}^{\infty}(\Lambda)$ given by (here $\omega \in \mathbb{R}$ ):

$$
q_{0}(\varphi, \psi):=\frac{1}{2}\langle(-i \nabla-\omega \mathbf{a}) \varphi,(-i \nabla-\omega \mathbf{a}) \psi\rangle
$$

By closing this form we generate a self-adjoint operator denoted by $H_{\Lambda}(\omega, 0)$, whose form core is $C_{0}^{\infty}(\Lambda)$, see e.g. [5, 30. For convenience we represent this operator as $H_{\Lambda}(\omega, 0)=\frac{1}{2}(-i \nabla-\omega \mathbf{a})^{2}$. If $\Lambda=\mathbb{R}^{3}$, the corresponding free magnetic operator is denoted by $H_{\infty}(\omega, 0)$. If $\mathbf{a}$ is smooth enough, then $H_{\Lambda}(\omega, 0)$ can be seen as the Friedrichs extension of $H_{\infty}(\omega, 0)$ restricted to $C_{0}^{\infty}(\Lambda)$.

The operator $H_{\Lambda}(\omega, 0)$ obeys the diamagnetic inequality [4, 30,

$$
\forall \varphi \in L^{2}(\Lambda), \forall \beta \geq 0, \quad\left|e^{-\beta H_{\Lambda}(\omega, 0)} \varphi\right| \leq e^{-\beta H_{\Lambda}(0,0)}|\varphi|
$$

We will work with electric potentials $V \in \mathcal{K}_{\text {loc }}\left(\mathbb{R}^{3}\right)$ which are $\mathbb{Z}^{3}$ periodic. We denote the restriction of $V$ to $\Lambda$ by the same symbol.

It is known that $V$ is infinitesimally form bounded to $H_{\Lambda}(0,0)$ [16, and implicitly to $H_{\Lambda}(\omega, 0)$; the last statement follows by using standard arguments involving the diamagnetic inequality (2.1) 
(see [5] and references herein). We conclude that the closure of the sesquilinear form defined on $C_{0}^{\infty}(\Lambda)$ and given by

$$
q_{V}(\varphi, \psi):=\frac{1}{2}\langle(-i \nabla-\omega \mathbf{a}) \varphi,(-i \nabla-\omega \mathbf{a}) \psi\rangle+\langle V \varphi, \psi\rangle
$$

will be symmetric, bounded from below and with the domain $Q\left(q_{V}\right)=Q\left(q_{0}\right)$. We denote by $H_{\Lambda}(\omega, V)$ its associated selfadjoint operator in $L^{2}(\Lambda)$.

The diamagnetic inequality (2.1) holds true if we replace the free operators by the perturbed one $H_{\Lambda}(\omega, V)$ and $H_{\Lambda}(0, V)$, see e.g. 223. This together with the min-max principle [28] imply:

$$
E_{0}(\omega):=\inf \sigma\left(H_{\Lambda}(\omega, V)\right) \geq e_{0}(\omega):=\inf \sigma\left(H_{\infty}(\omega, V)\right) \geq e_{0}(0)>-\infty .
$$

Remark 2.1. The operators $H_{\infty}(\omega, 0)$ and $H_{\Lambda}(\omega, 0)$ can be defined under weaker conditions on a see e.g. [5, 24, 25, 30] but the one imposed here will be necessary in what follows. When we work with a bounded $\Lambda$, the form domain of $H_{\Lambda}(\omega, V)$ will be $\mathcal{H}_{0}^{1}(\Lambda)$, independent of $\omega$ and $V$. If $\Lambda=\mathbb{R}^{3}$, then under our conditions on $V$ and a the operator $H_{\infty}(\omega, V)$ is selfadjoint and bounded from below having $C_{0}^{\infty}\left(\mathbb{R}^{3}\right)$ as a form core.

In the rest of the section we only consider the operator defined on the finite box. We allow $\omega \in \mathbb{C}$ and want to study the analyticity properties of the family $\left\{H_{\Lambda}(\omega, V), \omega \in \mathbb{C}\right\}$.

Proposition 2.2. Under conditions given above then $\left\{H_{\Lambda}(\omega, V), \omega \in \mathbb{C}\right\}$ is a type (B) entire family of operators. In particular $H_{\Lambda}(\omega, V), \omega \in \mathbb{C}$ are sectorial operators with sector:

$$
\mathcal{S}(\omega):=\left\{\xi \in \mathbb{C},|\operatorname{Im} \xi| \leq\left|\omega_{1}\right|\left(c_{1} \operatorname{Re} \xi+c_{2}\right), \operatorname{Re} \xi \in\left[c_{3},+\infty\right)\right\}
$$

where the constants $c_{1}, c_{2}, c_{3}$ satisfy: $0<c_{1}, c_{2}<\infty$ and $-\infty<-c_{2} / c_{1}<c_{3}<e_{0}$.

Proof. Denote by $T$ either $V$ or $\mathbf{a}^{2}$. Let $\omega_{0} \in \mathbb{R}$ and $\varphi \in Q\left(q_{V}\right),\|\varphi\|=1$. Then we know that for all $\sigma>0$ there exists $\sigma^{\prime}$ independent of $\omega_{0}$ such that

$$
|\langle T \varphi, \varphi\rangle| \leq \sigma\left\langle H_{\Lambda}\left(\omega_{0}, V\right) \varphi, \varphi\right\rangle+\sigma^{\prime}
$$

Let us show that the following two sesquilinear forms

$$
r_{1, \Lambda}\left(\omega_{0}\right):=\operatorname{Re}\left\{\mathbf{a} \cdot\left(i \nabla+\omega_{0} \mathbf{a}\right)\right\}, \quad r_{2, \Lambda}:=\frac{1}{2} \mathbf{a}^{2}
$$

are infinitesimally form bounded relatively to the form corresponding to $H_{\Lambda}\left(\omega_{0}, V\right)$.

Let $\omega_{0} \in \mathbb{R}$ and let $\varphi \in Q\left(q_{V}\right),\|\varphi\|=1$. The Cauchy-Schwarz inequality implies that for any $\alpha>0$ we have:

$$
\begin{array}{r}
\left|\left\langle\mathbf{a} \varphi,\left(i \nabla+\omega_{0} \mathbf{a}\right) \varphi\right\rangle\right| \leq \alpha\left\langle H_{\Lambda}\left(\omega_{0}, 0\right) \varphi, \varphi\right\rangle+\alpha^{-1}\left(\mathbf{a}^{2} \varphi, \varphi\right) \\
\leq \alpha\left\langle H_{\Lambda}\left(\omega_{0}, V\right) \varphi, \varphi\right\rangle+\alpha|\langle V \varphi, \varphi\rangle|+\alpha^{-1}\left\langle\mathbf{a}^{2} \varphi, \varphi\right\rangle
\end{array}
$$

In view of (2.3), then for all $\vartheta>0$ there exists $\vartheta^{\prime}>0$ both $\omega$-independent such that

$$
\left|\left\langle\mathbf{a} \varphi,\left(i \nabla+\omega_{0} \mathbf{a}\right) \varphi\right\rangle\right| \leq \vartheta\left\langle H_{\Lambda}\left(\omega_{0}, V\right) \varphi, \varphi\right\rangle+\vartheta^{\prime}
$$

This implies that the form $r_{1, \Lambda}\left(\omega_{0}\right)$ is bounded when restricted to the form domain of $H_{\Lambda}\left(\omega_{0}, V\right)$ and moreover, it generates an operator with zero relative form bound. This property also holds for the form $r_{2, \Lambda}$.

Now if $\omega \in \mathbb{C}$, denote $d \omega:=\omega-\omega_{0}$ and observe that we have in the form sense:

$$
r_{\Lambda}\left(\omega_{0}, \omega\right):=d \omega r_{1, \Lambda}\left(\omega_{0}\right)+d \omega^{2} r_{2, \Lambda}, \quad H_{\Lambda}(\omega, V)=H_{\Lambda}\left(\omega_{0}, V\right)+r_{\Lambda}\left(\omega_{0}, \omega\right)
$$

We conclude that the form domain of $H_{\Lambda}(\omega, V)$ is independent of $\omega: \mathcal{Q}\left(H_{\Lambda}(\omega, V)\right)=\mathcal{Q}\left(H_{\Lambda}(0, V)\right)$. Notice that (2.7) can be extended for $\omega_{0}, \omega \in \mathbb{C}$. We will now show that $\left\{H_{\Lambda}(\omega, V), \omega \in \mathbb{C}\right\}$ is 
a family of $m$-sectorial operators. Both properties ensure that $\left\{H_{\Lambda}(\omega, V), \omega \in \mathbb{C}\right\}$ is an analytic family of type (B) (see e.g. [26]).

Fix $\omega \in \mathbb{C}$ with $\operatorname{Re} \omega=\omega_{0}, \operatorname{Im} \omega=\omega_{1}$ and let $\varphi \in \mathcal{Q}\left(H_{\Lambda}\left(\omega_{0}, V\right)\right),\|\varphi\|=1$. Using (2.3), we conclude that for all $\sigma>0$ small enough such that $\sigma \omega_{1}^{2} \leq 1$ there exists $\sigma^{\prime}$ such that:

$$
\operatorname{Re}\left\langle H_{\Lambda}(\omega, V) \varphi, \varphi\right\rangle=\left\langle H_{\Lambda}\left(\omega_{0}, V\right) \varphi, \varphi\right\rangle-\frac{\omega_{1}^{2}}{2}\left\langle\mathbf{a}^{2} \varphi, \varphi\right\rangle \geq\left(1-\sigma \omega_{1}^{2} / 2\right)\left\langle H_{\Lambda}\left(\omega_{0}, V\right) \varphi, \varphi\right\rangle-\frac{\omega_{1}^{2}}{2} \sigma^{\prime}
$$

On the other hand, from (2.6) we conclude that there exist two constants $\vartheta, \vartheta^{\prime}>0$ such that

$$
\left|\operatorname{Im}\left\langle H_{\Lambda}(\omega, V) \varphi, \varphi\right\rangle\right|=\left|\omega_{1} \operatorname{Re}\left\langle\mathbf{a} \varphi,\left(i \nabla+\omega_{0} \mathbf{a}\right) \varphi\right\rangle\right| \leq\left|\omega_{1}\right|\left(\vartheta\left\langle H_{\Lambda}\left(\omega_{0}, V\right) \varphi, \varphi\right\rangle+\vartheta^{\prime}\right)
$$

Let $\Theta\left(H_{\Lambda}(\omega, V)\right)$ be the numerical range of $H_{\Lambda}(\omega, V)$. Then from (2.8) and (2.9) we obtain that both $\Theta\left(H_{\Lambda}(\omega, V)\right)$ and $\sigma\left(H_{\Lambda}(\omega, V)\right)$ are included in the sector (2.2).

Remark 2.3. (i). Note that $c_{1}, c_{2}, c_{3}$ in (2.2) depend implicitly on $\omega$ through the condition $\sigma \omega_{1}^{2} \leq 1$. If $\omega_{1}$ is small enough, then these constants can be chosen to be $\omega$ independent. Moreover, let $\omega_{0} \in \mathbb{R}$ and $\omega \in \mathbb{C}$ such that $|d \omega|$ is small enough. Then for all $\varphi \in \mathcal{Q}\left(H_{\Lambda}(\omega, V)\right)$, $\|\varphi\|=1$

$$
\operatorname{Re}\left\langle H_{\Lambda}(\omega, V) \varphi, \varphi\right\rangle \geq c_{3} \geq e_{0}\left(\omega_{0}\right)+\mathcal{O}(|d \omega|) .
$$

(ii). Let $\omega \in \mathbb{C}$. From [26] we know that if $\xi \notin \mathcal{S}(\omega),\left\|\left(H_{\Lambda}(\omega, V)-\xi\right)^{-1}\right\| \leq \frac{1}{d(\xi, \mathcal{S})}$. Hence put $\gamma(\omega)=\gamma:=-c_{2} / c_{1}$ and $\theta(\omega)=\theta:=\arctan \left(c\left|\omega_{1}\right|\right)$. For any $\delta>0$ introduce the sector

$$
\mathcal{S}_{\delta}(\omega):=\{\xi \in \mathbb{C},|\arg (\xi-\gamma)| \leq \theta+\delta\}
$$

Then there exists a constant $c_{\delta}>0$ such that for all $\xi \notin \mathcal{S}_{\delta}(\omega)$ we have

$$
\left\|\left(H_{\Lambda}(\omega, V)-\xi\right)^{-1}\right\| \leq \frac{c_{\delta}}{|\xi-\gamma|} .
$$

(iii). The operator $H_{\Lambda}(0, V)$ has compact resolvent (see e.g. [19, 27]). By standard arguments this also holds true for $H_{\Lambda}(\omega, V), \omega \in \mathbb{C}\left[26\right.$. Hence $H_{\Lambda}(\omega, V)$ has only discrete spectrum.

We are now interested in establishing Hilbert-Schmidt and trace norm estimates for powers of the resolvent at finite volume. Denote by $B_{1}$ the set of trace norm operators, and by $B_{2}$ the set of Hilbert-Schmidt operators defined on $L^{2}(\Lambda)$. We denote by $\|T\|_{1}$ and $\|T\|_{2}$ the trace norm, respectively the Hilbert-Schmidt norm of the operator $T$.

For $\beta>0$ and $\omega \in \mathbb{R}$, let

$$
W_{\Lambda}(\beta, \omega)=W_{\Lambda}(\beta, \omega, V):=e^{-\beta H_{\Lambda}(\omega, V)}
$$

be the strongly continuous semigroup associated to $H_{\Lambda}(\omega, V)$ on $L^{2}(\Lambda)$ see for example [26, 31] for the definition and general properties of a semigroup.

Lemma 2.4. There exist two positive constants $c_{0}$ and $C_{0}$ such that for every $\beta>0$ and $\omega \in \mathbb{R}$ we have that $W_{\Lambda}(\beta, \omega)$ is a positive trace class operators obeying:

$$
\left\|W_{\Lambda}(\beta, \omega)\right\|_{1}=\operatorname{Tr}_{\mathrm{L}^{2}(\Lambda)}\left\{\mathrm{W}_{\Lambda}(\beta, \omega)\right\} \leq \mathrm{c}_{0} \beta^{-3 / 2} \mathrm{e}^{\mathrm{C}_{0} \beta}|\Lambda| .
$$

Moreover, its Hilbert-Schmidt norm satisfies

$$
\left\|W_{\Lambda}(\beta, \omega)\right\|_{2} \leq c_{0} \beta^{-3 / 4} e^{C_{0} \beta}|\Lambda|^{\frac{1}{2}} .
$$


Proof. From [5] we know that the semigroup is an integral operator:

$$
\left(W_{\Lambda}(\beta, \omega) \varphi\right)(\mathbf{x})=\int_{\Lambda} G_{\Lambda}(\mathbf{x}, \mathbf{y}, \beta, \omega) \varphi(\mathbf{y}) d \mathbf{y}, \quad \varphi \in L^{2}(\Lambda) .
$$

Moreover the integral kernel $G_{\Lambda}$ is jointly continuous in $(\mathbf{x}, \mathbf{y}, \beta) \in \Lambda \times \Lambda \times \mathbb{R}_{+}^{*}$ and satisfies

$$
\left|G_{\Lambda}(\mathbf{x}, \mathbf{y}, \beta, \omega)\right| \leq c_{0} \beta^{-3 / 2} e^{C_{0} \beta} e^{\frac{-|\mathbf{x}-\mathbf{y}|^{2}}{4 \beta}},(\mathbf{x}, \mathbf{y}, \beta, \omega) \in \Lambda \times \Lambda \times \mathbb{R}_{+}^{*} \times \mathbb{R}
$$

for some positive constants $c_{0}, C_{0}$ which only depend on the potential $V$. This result comes from the monotonicity property of the semigroup and some generalized diamagnetic estimate [6, 30.

The proof of the lemma follows easily from (2.14). Note that the use of (2.14) is important in order to get the explicit $\beta$-dependance of quantities involved in the lemma.

We are now interested in obtaining similar estimates for powers of the resolvent. Let $\alpha>0$, $\omega \in \mathbb{R}, \xi_{0} \in \mathbb{C}, \operatorname{Re} \xi_{0}<e_{0}(\omega)$. As bounded operators on $L^{2}(\Lambda)$ we have [24, 30]

$$
\left(H_{\Lambda}(\omega, V)-\xi_{0}\right)^{-\alpha}=\frac{1}{\tilde{\gamma}(\alpha)} \int_{0}^{\infty} t^{\alpha-1} e^{\xi_{0} t} W_{\Lambda}(t, \omega) d t
$$

where $\tilde{\gamma}(\cdot)$ is the Euler gamma function. In particular from (2.12) and (2.13) for Re $\xi_{0}<0$ and $\left|\operatorname{Re} \xi_{0}\right|$ large enough there exists a constant $c>0$ independent of $\omega \in \mathbb{R}$ :

$$
\left\|\left(H_{\Lambda}(\omega, V)-\xi_{0}\right)^{-1}\right\|_{2} \leq c|\Lambda|^{\frac{1}{2}} \quad \text { and } \quad\left\|\left(H_{\Lambda}(\omega, V)-\xi_{0}\right)^{-2}\right\|_{1} \leq c|\Lambda| .
$$

\section{Proof of the main theorem.}

\section{$3.1 \omega$-analyticity of resolvents.}

The first technical result is the following:

Proposition 3.1. Let $\omega \in \mathbb{C}$ and $\xi \in \rho\left(H_{\Lambda}(\omega, V)\right)$. Then there exists a complex neighborhood $\mathcal{V}_{\xi}(\omega)$ of $\omega$ such that $\xi \in \rho\left(H_{\Lambda}\left(\omega^{\prime}, V\right)\right)$ and the operator valued function $\mathcal{V}_{\xi}(\omega) \ni \omega^{\prime} \mapsto\left(H_{\Lambda}\left(\omega^{\prime}, V\right)-\right.$ $\xi)^{-1}$ is $B_{2}$-analytic.

Proof. Let $\omega \in \mathbb{C}$. First we choose $\xi_{0}<0$ negative enough so that $\xi_{0} \in \rho\left(H_{\Lambda}(\omega, V)\right)$. Such a choice is possible because $H_{\Lambda}(\omega, V)$ is $m$-sectorial.

It is a well known fact [27] that since the perturbation $r_{\Lambda}(0, \omega)$ (see (2.7)) is relatively form bounded to $H_{\Lambda}(0, V)$ with zero bound then for $\xi_{0}<0$ with $\left|\xi_{0}\right|$ large enough there exists some complex neighborhood of $\omega$ denoted by $\nu(\omega)$ such that for all $\omega^{\prime} \in \nu(\omega)$ one has:

$$
\left\|\left(H_{\Lambda}(0, V)-\xi_{0}\right)^{-1 / 2} r_{\Lambda}\left(0, \omega^{\prime}\right)\left(H_{\Lambda}(0, V)-\xi_{0}\right)^{-1 / 2}\right\|<1 .
$$

Set $K\left(\xi_{0}, \omega^{\prime}\right):=\left(H_{\Lambda}(0, V)-\xi_{0}\right)^{-1 / 2} r_{\Lambda}\left(0, \omega^{\prime}\right)\left(H_{\Lambda}(0, V)-\xi_{0}\right)^{-1 / 2}$. From the estimate (3.1) we conclude that $\forall \omega^{\prime} \in \nu(\omega), \xi_{0} \in \rho\left(H_{\Lambda}\left(\omega^{\prime}, V\right)\right)$ and

$$
\left(H_{\Lambda}\left(\omega^{\prime}, V\right)-\xi_{0}\right)^{-1}=\left(H_{\Lambda}(0, V)-\xi_{0}\right)^{-1 / 2}\left(\mathbb{I}+K\left(\xi_{0}, \omega^{\prime}\right)\right)^{-1}\left(H_{\Lambda}(0, V)-\xi_{0}\right)^{-1 / 2} .
$$

holds in the bounded operator sense. And since $\omega^{\prime} \in \nu(\omega) \rightarrow K\left(\xi_{0}, \omega^{\prime}\right)$ is analytic, it follows that the bounded operators valued function $\omega^{\prime} \in \nu(\omega) \rightarrow\left(\mathbb{I}+K\left(\xi_{0}, \omega^{\prime}\right)\right)^{-1}$ is analytic too.

On the other hand, from (3.2) we have

$$
\left\|\left(H_{\Lambda}\left(\omega^{\prime}, V\right)-\xi_{0}\right)^{-1}\right\|_{2} \leq\left\|\left(\mathbb{I}+K\left(\xi_{0}, \omega^{\prime}\right)\right)^{-1}\right\|\left\|\left(H_{\Lambda}(0, V)-\xi_{0}\right)^{-1}\right\|_{2}
$$

which together with (2.16), it shows that $\omega^{\prime} \in \nu(\omega) \rightarrow\left(H_{\Lambda}\left(\omega^{\prime}, V\right)-\xi_{0}\right)^{-1}$ is a Hilbert-Schmidt family of operators in $\omega^{\prime} \in \nu(\omega)$. Now it is straightforward to prove the theorem for such a $\xi_{0}$. It remains to extend the $B_{2}$-analyticity property for any $\xi \in \rho\left(H_{\Lambda}(\omega, V)\right)$. 
Let $\xi_{0}$ as above and consider the first resolvent equation

$$
\left(H_{\Lambda}(\omega, V)-\xi\right)^{-1}=\left(H_{\Lambda}(\omega, V)-\xi_{0}\right)^{-1}+\left(\xi-\xi_{0}\right)\left(H_{\Lambda}(\omega, V)-\xi\right)^{-1}\left(H_{\Lambda}(\omega, V)-\xi_{0}\right)^{-1}
$$

Since there exists a bounded complex neighborhood $V_{\xi}(\omega)$ of $\omega$ such that the operator-valued function $V_{\xi}(\omega) \ni \omega^{\prime} \mapsto\left(H_{\Lambda}\left(\omega^{\prime}, V\right)-\xi\right)^{-1}$ is bounded-analytic, by standard arguments involving the bilateral ideal property of $B_{2}$, the operator-valued function $\omega^{\prime} \mapsto\left(H_{\Lambda}\left(\omega^{\prime}, V\right)-\xi\right)^{-1}\left(H_{\Lambda}\left(\omega^{\prime}, V\right)-\right.$ $\left.\xi_{0}\right)^{-1}$ is $B_{2}$-analytic on $V_{\xi}(\omega) \cap \nu(\omega)$. Now use (3.3) and the proof is over.

Corollary 3.2. Let $\omega \in \mathbb{C}$ and $\xi \in \rho\left(H_{\Lambda}(\omega, V)\right)$. Then there exists a neighborhood $\mathcal{V}_{\xi}(\omega)$ of $\omega$ such that the operator valued function $\mathcal{V}_{\xi}(\omega) \ni \omega^{\prime} \mapsto\left(H_{\Lambda}\left(\omega^{\prime}, V\right)-\xi\right)^{-2}$ is $B_{1}$-analytic.

Proof. From Proposition 3.1 we have that $\left(H_{\Lambda}(\omega, V)-\xi\right)^{-2}$ is a product of two Hilbert-Schmidt operators. Thus $\left(H_{\Lambda}(\omega, V)-\xi\right)^{-2}$ is trace class. Then by a direct calculus we can check the statement of the corollary.

Now we consider $W_{\Lambda}(\beta, \omega), \omega \in \mathbb{R}$. We want to extend $W_{\Lambda}(\beta, \omega)$ to complex $\omega$ 's and in trace class sense. We will use the fact that the operator $H_{\Lambda}(\omega, V)$ is $m$-sectorial:

Corollary 3.3. Let $\beta>0$. The family $\left\{W_{\Lambda}(\beta, \omega), \omega \in \mathbb{R}\right\}$ can be extended to a $B_{1}$ entire family of operators.

Proof. Let $\beta>0, \omega \in \mathbb{C}$. Consider the curve in $\mathbb{C}$ given by $\Gamma:=\left\{\xi \in \mathbb{C}:\left|\arg \left(\xi-\gamma^{\prime}\right)\right|=\theta+\epsilon\right\}$ where $\gamma^{\prime}, \epsilon$ are chosen such that $\gamma-\gamma^{\prime}=1$ and $\theta+\epsilon<\frac{\pi}{2}$. Here $\gamma, \theta$ are given by the Remark 2.3 ii). The curve $\Gamma$ encloses the spectrum of $H_{\Lambda}\left(\omega^{\prime}, V\right)$ for all $\omega^{\prime}$ in a neighborhood of $\omega, \nu(\omega)$. From the Dunford functional calculus [17, the following relation holds in terms of bounded operators:

$$
W_{\Lambda}\left(\beta, \omega^{\prime}\right):=\frac{i}{2 \pi} \int_{\Gamma} d \xi e^{-\beta \xi}\left(H_{\Lambda}\left(\omega^{\prime}, V\right)-\xi\right)^{-1}, \quad \omega^{\prime} \in \nu(\omega)
$$

This shows that the semigroup can be extended to a bounded operator for every complex $\omega^{\prime}$. We now want to show that this formula defines in fact a trace class operator. Choose $\xi_{0} \in \mathbb{C}$ with $\operatorname{Re} \xi_{0}<0$ and $\left|\operatorname{Re} \xi_{0}\right|$ large enough so that $\xi_{0} \in \rho\left(H_{\Lambda}\left(\omega^{\prime}, V\right)\right)$ for any $\omega^{\prime} \in \nu(\omega)$. Using twice the resolvent formula (3.3) in (3.4) we obtain the identity:

$$
W_{\Lambda}\left(\beta, \omega^{\prime}\right)=\frac{i}{2 \pi} \int_{\Gamma} d \xi e^{-\beta \xi}\left(\xi-\xi_{0}\right)^{2}\left(H_{\Lambda}\left(\omega^{\prime}, V\right)-\xi\right)^{-1}\left(H_{\Lambda}\left(\omega^{\prime}, V\right)-\xi_{0}\right)^{-2}, \quad \omega^{\prime} \in \nu(\omega)
$$

From the choice of $\Gamma$, the bounded operator valued function $\nu(\omega) \ni \omega^{\prime} \rightarrow\left(H_{\Lambda}\left(\omega^{\prime}, V\right)-\xi\right)^{-1}$ is analytic for all $\xi \in \Gamma$, and all norm bounds are uniform in $\xi \in \Gamma$. Moreover, from (2.11) we conclude that there exists a constant $C>0$ such that $\left\|e^{-\beta \xi}\left(\xi-\xi_{0}\right)^{2}\left(H_{\Lambda}\left(\omega^{\prime}, V\right)-\xi\right)^{-1}\right\| \leq C|\operatorname{Re} \xi|^{2} e^{-\beta \operatorname{Re} \xi}$. Therefore

$$
\nu(\omega) \ni \omega^{\prime} \rightarrow \int_{\Gamma} d \xi e^{-\beta \xi}\left(\xi-\xi_{0}\right)^{2}\left(H_{\Lambda}\left(\omega^{\prime}, V\right)-\xi\right)^{-1}
$$

is bounded analytic too. Hence from Corollary 3.2 and (3.5) the operators valued function $\nu(\omega) \ni$ $\omega^{\prime} \rightarrow W_{\Lambda}\left(\beta, \omega^{\prime}\right)$ is $B_{1}$-analytic. Thus $W_{\Lambda}(\beta, \cdot)$ is $B_{1}$-entire. 


\section{$3.2 \omega$-analyticity of the pressure.}

Let $\beta>0, \omega \in \mathbb{R}$ and $z \in D_{\epsilon}\left(e_{0}\right) \cap \mathbb{R}$. Define

$$
\left[e_{0}(\omega), \infty\right) \ni \xi \mapsto \ln \left(1+\epsilon z e^{-\beta \xi}\right)
$$

We have that the map $(z, \xi) \mapsto \ln \left(1+\epsilon z e^{-\beta \xi}\right)$ is jointly analytic in

$$
\left\{(z, \xi) \in \mathbb{C} \times \mathbb{C}:|z| e^{-\beta \operatorname{Re} \xi}<1\right\}
$$

but this is not sufficient and we also need to control the region in which $\operatorname{Re} \xi$ is close to the bottom of the spectrum. Let $\beta>0, \omega \in \mathbb{R},-\infty<e_{0}^{\prime} \leq e_{0}=e_{0}(\omega)$ and consider the domains $D_{\epsilon}\left(e_{0}^{\prime}\right)$ defined as in (1.1) but with $e_{0}^{\prime}$ instead $e_{0}$. Then:

Lemma 3.4. Let $\beta>0, \omega \in \mathbb{R}$ and $-\infty<e_{0}^{\prime} \leq e_{0}$. For each compact $K \subset D_{\epsilon}\left(e_{0}^{\prime}\right)$ there exists $\eta_{K}>0$ such that $(z, \xi) \mapsto \ln \left(1+\epsilon z e^{-\beta \xi}\right)$ is jointly analytic in

$$
K \times\left\{\xi \in \mathbb{C}: \operatorname{Im} \xi \in\left(-\frac{\eta_{K}}{\beta}, \frac{\eta_{K}}{\beta}\right), \quad \operatorname{Re} \xi \in\left[e_{0}^{\prime}, \infty\right)\right\}
$$

If $K^{\prime}$ is a compact subset such that $K^{\prime} \subset K$ then $\eta_{K}^{\prime}>\eta_{K}$.

Proof. We first deal with the Bose case. Here $\mathcal{B}(r)$ is an open ball in $\mathbb{C}$ centered at the origin having radius $r>0$. Obviously $(z, \xi) \mapsto \ln \left(1+\epsilon z e^{-\beta \xi}\right)$ is a jointly analytic function in $\operatorname{Re} \xi \in$ $\left[e_{0}^{\prime}(\omega), \infty\right), z \in \mathcal{B}\left(e^{\beta e_{0}^{\prime}}\right)$. Let $K$ be a compact of $D_{-1}\left(e_{0}^{\prime}\right)$ and denote by $\tilde{K}=K \backslash \mathcal{B}\left(e^{\beta e_{0}^{\prime}}\right)$. Let

$$
\theta_{m}:=\inf \{\arg (z), z \in \tilde{K}\}, \quad \theta_{M}:=\sup \{\arg (z), z \in \tilde{K}\}
$$

Because $\operatorname{dist}\left(\tilde{K},\left[e^{\beta e_{0}^{\prime}}, \infty\right)\right)>0$, then $0<\theta_{m} \leq \theta_{M}<2 \pi$. We set $\eta_{K}:=\frac{1}{2} \inf \left\{\theta_{m}, 2 \pi-\theta_{M}\right\}$. Clearly for $z \in \tilde{K}$ and $\operatorname{Im} \xi \in\left[-\frac{\eta_{K}}{\beta}, \frac{\eta_{K}}{\beta}\right], 0<\frac{\theta_{m}}{2} \leq \arg z-\beta \operatorname{Im} \xi \leq \pi+\frac{\theta_{M}}{2}<2 \pi$ then $\operatorname{Im}\left(1-z e^{-\beta \xi}\right)=0$ iff $\arg z-\beta \operatorname{Im} \xi=\pi$ but in this last case $\operatorname{Re}\left(1-z e^{-\beta \xi}\right)>0$.

For the Fermi case we get the lemma following the same arguments as above. Let $K$ be a compact of $D_{+1}\left(e_{0}^{\prime}\right)$ and denote by $\tilde{K}=K \backslash \mathcal{B}\left(e^{\beta e_{0}^{\prime}}\right)$. Let

$$
\theta_{m}:=\sup \{\arg (z), z \in \tilde{K}, \arg (z) \geq 0\}, \quad \theta_{M}:=\inf \{\arg (z), z \in \tilde{K}, \arg (z)<0\}
$$

We set $\eta_{K}:=\frac{1}{2} \inf \left\{\pi-\theta_{m}, \pi+\theta_{M}\right\}$. Clearly for $z \in \tilde{K}$ and $\operatorname{Im} \xi \in\left[-\frac{\eta_{K}}{\beta}, \frac{\eta_{K}}{\beta}\right],-\pi<-\frac{\pi}{2}+\frac{\theta_{M}}{2} \leq$ $\arg z-\beta \operatorname{Im} \xi \leq \frac{\pi}{2}+\frac{\theta_{m}}{2}<\pi$ then $\operatorname{Im}\left(1+z e^{-\beta \xi}\right)=0$ iff $\arg z-\beta \operatorname{Im} \xi=0$ but in this last case $\operatorname{Re}\left(1+z e^{-\beta \xi}\right)>0$.

Proposition 3.5. Let $\beta>0, \omega_{0} \in \mathbb{R}$ and $K \subset D_{\epsilon}\left(e_{0}\left(\omega_{0}\right)\right)$ a compact subset. Then there exists a complex neighborhood $\mathcal{V}\left(\omega_{0}\right)$ of $\omega_{0}$ such that for any $z \in K$, the pressure is an analytic function w.r.t. $\omega$ in $\mathcal{V}\left(\omega_{0}\right)$.

Proof. Let $\omega_{0} \in \mathbb{R}$, and $K \subset D_{\epsilon}\left(e_{0}\right), e_{0}=e_{0}\left(\omega_{0}\right)$ be a compact subset.Then there exists $e_{0}^{\prime}$ satisfying $-\infty<e_{0}^{\prime}<e_{0}$ such that $K \subset D_{\epsilon}\left(e_{0}^{\prime}\right)$. Consider now the following positively oriented contour defined by

$$
\begin{gathered}
\Gamma_{K}:=\left\{\operatorname{Re} \xi=e_{0}^{\prime}, \operatorname{Im} \xi \in\left[-\frac{\eta_{K}}{2 \beta}, \frac{\eta_{K}}{2 \beta}\right]\right\} \cup\left\{\operatorname{Re} \xi \in\left[e_{0}^{\prime}, \xi_{K}\right),|\operatorname{Im} \xi|=\frac{\eta_{K}}{2 \beta}\right\} \cup \\
\left\{\operatorname{Re} \xi \geq \xi_{K}, \arg \left(\xi-\xi_{K} \mp i \frac{\eta_{K}}{2 \beta}\right)=\mp \frac{\pi}{4}\right\}
\end{gathered}
$$


where $\eta_{K}>0$ is given by (3.7); $\xi_{K}$ is chosen so that $\xi_{K}>e_{0}$ and satisfies the condition (3.6) i.e.

$$
\sup _{z \in K}\{|z|\} e^{-\beta \operatorname{Re} \xi}<1 \quad \text { if } \quad \operatorname{Re} \xi>\xi_{\mathrm{K}}
$$

Recall the domain of analyticity of $\xi \mapsto \ln \left(1+\epsilon z e^{-\beta \xi}\right)$ defined by lemma 3.4 knowing (3.6). Then $\Gamma_{K}$ is enclosed in this domain of analyticity.

Let $\mathcal{B}\left(\omega_{0}, r\right)$ be an open ball in $\mathbb{C}$ centered at $\omega_{0}$ and radius $r>0$. If $r$ is small enough then for $\omega \in \mathcal{B}\left(\omega_{0}, r\right)$, the spectrum of $H_{\Lambda}(\omega, V)$ as well as the sector $\mathcal{S}(\omega)$ defined in (2.10) for $\operatorname{Re} \xi>\xi_{K}$ together lie inside $\Gamma_{K}$. To see this we use the Remark $\left.2.3 \mathrm{i}\right)$.

For $\beta>0, z \in K$ and $\omega \in \mathcal{B}\left(\omega_{0}, r\right)$ consider the following Dunford integral operator [17]

$$
I(\beta, z, \omega):=\frac{i}{2 \pi} \int_{\Gamma_{K}} \mathrm{~d} \xi \ln \left(1+\epsilon z e^{-\beta \xi}\right)\left(H_{\Lambda}(\omega, V)-\xi\right)^{-1}
$$

The above integral converges and defines a bounded operator due to the exponential decay of $\ln \left(1+\epsilon z e^{-\beta \xi}\right)$ in $\operatorname{Re} \xi$ and because of (2.11).

Again here the choice of the contour implies that if $r$ is small enough then for each $\xi \in \Gamma_{K}$ the bounded operator valued function $\mathcal{B}\left(\omega_{0}, r\right) \ni \omega \mapsto\left(H_{\Lambda}(\omega, V)-\xi\right)^{-1}$ is analytic. Therefore for $r$ small enough $\left\{I(\beta, z, \omega), \omega \in \mathcal{B}\left(\omega_{0}, r\right)\right\}$ is an analytic family of bounded operators in $L^{2}(\Lambda)$. By analytic continuation we conclude that

$$
I(\beta, z, \omega)=\ln \left(\mathbb{I}+\epsilon z W_{\Lambda}(\beta, \omega)\right)
$$

for all $\omega \in \mathcal{B}\left(\omega_{0}, r\right)$ because the equality holds for real $\omega$.

Now choose a $\xi_{0}$ with a very negative $\operatorname{Re} \xi_{0}$. Then we get:

$$
\ln \left(\mathbb{I}+\epsilon z W_{\Lambda}(\beta, \omega)\right)=\left(\frac{i}{2 \pi} \int_{\Gamma_{K}} \mathrm{~d} \xi\left(\xi-\xi_{0}\right)^{2} \ln \left(1+\epsilon z e^{-\beta \xi}\right)\left(H_{\Lambda}(\omega, V)-\xi\right)^{-1}\right)\left(H_{\Lambda}(\omega, V)-\xi_{0}\right)^{-2}
$$

This implies that if $r$ is small enough, the family $\left\{I(\beta, z, \omega), \omega \in \mathcal{B}\left(\omega_{0}, r\right)\right\}$ is also analytic in the trace class topology. The proof is over.

\subsection{Proof of the analyticity w.r.t $\omega$ and $z$.}

Recall that the Hartog theorem [20, implies the joint analyticity once we know the analyticity w.r.t. each variable separately.

Put $\mathcal{V}:=\bigcup_{\omega_{0} \in \mathbb{R}} \mathcal{V}\left(\omega_{0}\right)$. Then from the Proposition 3.5 for any $z \in K$ the pressure is an analytic function with respect to $\omega$ in $\mathcal{V}$. This is the first thing we need in order to apply the Hartog theorem.

Now let $\beta>0$ and $K$ as in the theorem. We want to show that there exists a neighborhood of the real axis $\mathcal{N}$ such that for any $\omega \in \mathcal{N}$, the function $K \ni z \mapsto P_{\Lambda}(\beta, \omega, z, \epsilon)$ is analytic.

We use formula (3.9) but with $e_{0}^{\prime}<e_{0}(0)$ in the definition (3.8) of $\Gamma_{K}$. The only thing we have to show is that for $\omega \in \mathbb{C}$, $\operatorname{Im} \omega$ small enough the $B_{1}$-operator valued function $\Gamma_{K} \ni \xi \rightarrow$ $\left(H_{\Lambda}(\omega)-\xi\right)^{-1}\left(H_{\Lambda}(\omega)-\xi_{0}\right)^{-2}$ is uniformly bounded for Re $\xi$ large enough. But this is true since (2.11) implies that $\xi \in \Gamma_{K} \rightarrow\left\|\left(H_{\Lambda}(\omega)-\xi\right)^{-1}\right\|$ is uniformly bounded for Re $\xi$ large enough and we know that $\left(H_{\Lambda}(\omega)-\xi_{0}\right)^{-2} \in B_{2}$. The proof is over.

For $\beta>0, \omega_{0} \in \mathbb{R}$ and $z \in D_{\epsilon}\left(e_{0}\right)$, the grand canonical generalized susceptibilities at finite volume are defined by

$$
\chi_{\Lambda}^{N}\left(\beta, \omega_{0}, z, \epsilon\right):=\left(\frac{e}{c}\right)^{N} \frac{\partial^{N} P_{\Lambda}}{\partial \omega^{N}}\left(\beta, \omega_{0}, z, \epsilon\right), \quad N \in \mathbb{N}^{*}
$$


By Proposition 3.5, $\chi_{\Lambda}^{N}\left(\beta, \omega_{0}, z, \epsilon\right)$ are well defined. In the physical literature (see e.g. [22]), the cases $N=1$ and $N=2$ correspond respectively to the grand canonical magnetization and magnetic susceptibility per unit volume. Moreover

Corollary 3.6. Let $\beta>0$ and $N \geq 1$. For each open set $K$ with the property that $\bar{K}$ is compact and $\bar{K} \subset \mathbf{D}_{\epsilon}$, there exists a complex neighborhood $\mathcal{N}$ of the real axis such that $\mathcal{N} \times K \ni(\omega, z) \mapsto$ $\chi_{\Lambda}^{N}(\beta, \omega, z, \epsilon)$ is analytic.

\subsection{The thermodynamic limit.}

Now assume that the domain $\Lambda$ is obtained by dilating a given set $\Lambda_{1} \subset \mathbb{R}^{3}$ which is supposed to be bounded, open, simply connected and with smooth boundary. More precisely:

$$
\Lambda_{L}:=\left\{\mathbf{x} \in \mathbb{R}^{3}: \mathbf{x} / L \in \Lambda_{1}, \quad L>1\right\}
$$

Assume that the electric potential $V$ belongs to $\mathcal{K}_{l o c}$ and is $\mathbb{Z}^{3}$ periodic, and denote its elementary cell with $\Omega$. We also assume that the magnetic potential a can be written as $\mathbf{a}_{c}+\mathbf{a}_{p}$, where $\mathbf{a}_{c}$ is the symmetric gauge given by a constant magnetic field (thus has a linear growth), while $\left|\mathbf{a}_{p}\right|^{2}$ belongs to $\mathcal{K}_{l o c}$ and is $\mathbb{Z}^{3}$ periodic. Let $\chi_{\Omega}$ denote the characteristic function of the elementary cell.

Let $\omega \in \mathbb{R}$. Introduce the integrated density of states (IDS) defined as the following thermodynamic limit if it exists. Let $E \in \mathbb{R}$ and $N(E)$ denotes the number of eigenvalues of the operator $H_{\Lambda}(\omega, V)$ smaller than $E$ counting with their multiplicity.

$$
\rho(E):=\lim _{L \rightarrow \infty} \frac{N(E)}{\left|\Lambda_{L}\right|}
$$

Let $P(I)$ the spectral projector associated with the operator $H_{\infty}(\omega, V)$ on the interval $I$. Then we have [18, 24]

Proposition 3.7. Under the condition stated above then the IDS of $H_{\infty}(\omega, V)$ exists and for almost all $E \in \mathbb{R}$

$$
\rho(E):=\frac{1}{|\Omega|} \operatorname{Tr}_{\mathrm{L}^{2}\left(\mathbb{R}^{3}\right)}\left(\chi_{\Omega} \mathrm{P}(\mathrm{E})\right)
$$

where $\mathrm{P}(\mathrm{E}):=\mathrm{P}(-\infty, \mathrm{E}]$.

This last proposition allows us to compute the thermodynamic limit of the pressure. Recall that we have shown that the pressure at finite volume for $\omega \in \mathbb{R}, \beta>0$, and $z \in K$ where $K$ is a compact subset of $D_{\epsilon}\left(e_{0}\right)$ can be expressed as:

$$
P_{\Lambda_{L}}(\beta, \omega, z, \epsilon)=\frac{i \epsilon}{2 \beta \pi\left|\Lambda_{L}\right|} \operatorname{Tr}_{L^{2}\left(\Lambda_{L}\right)} \int_{\Gamma_{K}} \mathrm{~d} \xi \ln \left(1+\epsilon z e^{-\beta \xi}\right)\left(H_{\Lambda_{L}}(\omega, V)-\xi\right)^{-1}
$$

Let $\omega$ real, define [15] :

$$
P_{\infty}(\beta, \omega, z, \epsilon)=\frac{i \epsilon}{2 \beta \pi|\Omega|} \operatorname{Tr}_{L^{2}\left(\mathbb{R}^{3}\right)} \int_{\Gamma_{K}} \mathrm{~d} \xi \ln \left(1+\epsilon z e^{-\beta \xi}\right) \chi_{\Omega}\left(H_{\infty}(\omega, V)-\xi\right)^{-1} \chi_{\Omega}
$$

The above integral defines a trace class operator on $L^{2}\left(\mathbb{R}^{3}\right)$ because after a use of the resolvent identity we can change the integrand into:

$$
\left(\xi-\xi_{0}\right) \ln \left(1+\epsilon z e^{-\beta \xi}\right) \chi_{\Omega}\left(H_{\infty}(\omega, V)-\xi\right)^{-1}\left(H_{\infty}(\omega, V)-\xi_{0}\right)^{-1} \chi_{\Omega}
$$

where $\xi_{0}$ is some fixed and negative enough number. Using the Laplace transform and the properties of the semigroup $e^{-t H_{\infty}}$ one can prove that $\chi_{\Omega}\left(H_{\infty}(\omega, V)-\xi\right)^{-1}$ and $\left(H_{\infty}(\omega, V)-\xi_{0}\right)^{-1} \chi_{\Omega}$ are Hilbert-Schmidt operators whose norms grow polynomially with $\operatorname{Re} \xi$.

Then we have: 
Theorem 3.8. Let $\omega \in \mathbb{R}, \beta>0$ and $K \subset D_{\epsilon}\left(e_{0}\right)$ a compact set. Under the same condition as above then uniformly in $z \in K$

$$
\lim _{L \rightarrow \infty} P_{\Lambda_{L}}(\beta, \omega, z, \epsilon)=P_{\infty}(\beta, \omega, z, \epsilon) .
$$

Proof. Define $(\xi, z) \mapsto f(\xi, z)=f(\xi, \beta, z, \epsilon):=\ln \left(1+\epsilon z e^{-\beta \xi}\right)$. First recall the well known formula (see e.g. [11) which holds if the IDS exists

$$
\lim _{L \rightarrow \infty} P_{\Lambda_{L}}(\beta, \omega, z, \epsilon)=P_{\infty}(\beta, \omega, z, \epsilon) ; \quad P_{\infty}(\beta, \omega, z, \epsilon)=-\frac{\epsilon}{\beta} \int_{\mathbb{R}} f_{\xi}^{\prime}(\xi, z) \rho(\xi) d \xi
$$

Then by using Proposition 3.7 and the fact that $\left\{\chi_{\Omega} \mathrm{P}(\mathrm{E}), \mathrm{E} \in \mathbb{R}\right\}$ is a family of trace class operators we get

$$
P_{\infty}(\beta, \omega, z, \epsilon)=-\frac{\epsilon}{\beta|\Omega|} \int_{\mathbb{R}} f_{\xi}^{\prime}(\xi, z) \operatorname{Tr}_{L^{2}\left(\mathbb{R}^{3}\right)}\left(\chi_{\Omega} \mathrm{P}(\xi)\right) \mathrm{d} \xi=\frac{\epsilon}{\beta|\Omega|} \operatorname{Tr}_{\mathrm{L}^{2}\left(\mathbb{R}^{3}\right)}\left(\chi_{\Omega} \int_{\mathbb{R}} \mathrm{f}(\xi, \mathrm{z}) \mathrm{dP}(\xi)\right) .
$$

So by the spectral theorem

$$
P_{\infty}(\beta, \omega, z, \epsilon)=\frac{\epsilon}{\beta|\Omega|} \operatorname{Tr}_{L^{2}\left(\mathbb{R}^{3}\right)}\left(\chi_{\Omega} f\left(H_{\infty}, z\right)\right)
$$

and then by using again the Dunford integral representation [17 the theorem follows.

The fact that $\omega$ must be real is an important ingredient of the proof of (3.14) where one extensively uses the gauge invariance of the operators and the fact that $H_{\infty}$ commutes with the magnetic translations generated by $\mathbb{Z}^{3}$. It is shown in 29 that if $\mathbf{a}_{c}=0$ i.e. the magnetic vector potential is periodic, then the limit in (3.14) holds true for every $\omega$ is a small ball around every $\omega_{0} \in \mathbb{R}$, provided that $z$ and $\beta$ are fixed. The explanation is that the analyticity ball in $\omega$ which we have constructed for each $P_{\Lambda_{L}}$ would be independent of $L$. If $\mathcal{C}_{r}\left(\omega_{0}\right)$ denotes the positively oriented circle with radius $r$ and center at $\omega_{0}$, then for any real $\omega$ inside $\mathcal{C}_{r}\left(\omega_{0}\right)$ and for $r$ small enough we can write:

$$
P_{\Lambda_{L}}(\omega)=\frac{1}{2 \pi i} \int_{\mathcal{C}_{r}\left(\omega_{0}\right)} \frac{P_{\Lambda_{L}}\left(\omega^{\prime}\right)}{\omega^{\prime}-\omega} d \omega^{\prime}, \quad \chi_{\Lambda_{L}}^{N}(\omega)=\frac{N !}{2 \pi i} \int_{\mathcal{C}_{r}\left(\omega_{0}\right)} \frac{P_{\Lambda_{L}}\left(\omega^{\prime}\right)}{\left(\omega^{\prime}-\omega\right)^{N+1}} d \omega^{\prime}
$$

The last integral representation of $\chi_{\Lambda_{L}}^{N}(\omega)$ tells us that if the pressure admits the thermodynamic limit, the same property holds true for all generalized susceptibilities. Thus the existence of the thermodynamic limit of the generalized susceptibilities follows easily if there is no linear growth in the magnetic potential generated by the magnetic field.

If $\mathbf{a}_{c}$ is not zero, then the above argument breaks down because $r$ (the analyticity radius in $\omega$ of $P_{\Lambda_{L}}$ ) goes to zero with $L$. In fact one cannot hope to prove in general that $P_{\infty}$ is real analytic in $\omega$, although one can prove that it is smooth in $\omega \in \mathbb{R}$. In order to achieve that, one needs to use the magnetic perturbation theory methods developed in [15, 7, 8, 9,. Complete proofs will be given in 29.

\subsection{The canonical ensemble.}

Let $\rho_{0}>0$ be the density of particles. The number of particles in the finite box $\Lambda$ is $N_{\Lambda}=\rho_{0}|\Lambda|$. For $\beta>0, \omega_{0} \in \mathbb{R}$ and $\rho_{0}>0$ fixed, define the finite volume Helmholtz free energy (see [22]) as

$$
f_{\Lambda}\left(\beta, \rho_{0}, \omega_{0}, \epsilon\right):=-\frac{1}{\beta} \ln \left(Z_{\Lambda}\left(\beta, \rho_{0}, \omega_{0}, \epsilon\right)\right)
$$

where $Z_{\Lambda}\left(\beta, \rho_{0}, \omega_{0}\right)>0$ stands for the canonical partition function.

As a consequence of Theorem 1.1, we have: 
Corollary 3.9. Let $\beta>0$ and $\rho_{0}>0$. Then there exists a complex neighborhood of the real axis $\mathcal{M}$ such that the the Helmholtz free energy $\mathcal{M} \ni \omega \mapsto f_{\Lambda}\left(\beta, \rho_{0}, \omega, \epsilon\right)$ is analytic.

Proof. For all $\omega_{0} \in \mathbb{R}$, the canonical partition function is related to the grand-canonical pressure by (see [13)

$$
Z_{\Lambda}\left(\beta, \rho_{0}, \omega_{0}, \epsilon\right):=\frac{1}{2 i \pi} \int_{\mathcal{C}} d z \frac{1}{z}\left[\frac{\exp \left(\frac{\beta}{\rho_{0}} P_{\Lambda}\left(\beta, \omega_{0}, z, \epsilon\right)\right)}{z}\right]^{N_{\Lambda}}
$$

where $\mathcal{C}$ is a closed contour around 0 and included in the analyticity domain $\mathbf{D}_{\epsilon}$ of the function $z \rightarrow P_{\Lambda}\left(\beta, \omega_{0}, z, \epsilon\right)$. From Theorem 1.1. there exists a complex neighborhood $\mathcal{M}^{\prime}$ of the real axis such that $\mathcal{M}^{\prime} \ni \omega \mapsto Z_{\Lambda}\left(\beta, \rho_{0}, \omega, \epsilon\right)$ is analytic. Since $Z_{\Lambda}\left(\beta, \rho_{0}, \omega_{0}, \epsilon\right)>0$ for all $\omega_{0} \in \mathbb{R}$, then by a continuity argument, there exists a complex neighborhood $\mathcal{M}$ of the real axis such that for all $\omega \in \mathcal{M}, \operatorname{Re} Z_{\Lambda}\left(\beta, \rho_{0}, \omega, \epsilon\right)>0$. Then the corollary follows.

For $\beta>0, \rho_{0}>0$ and $\omega_{0} \in \mathbb{R}$, the canonical generalized susceptibilities at finite volume are defined by

$$
m_{\Lambda}^{N}\left(\beta, \rho_{0}, \omega_{0}, \epsilon\right):=-\frac{1}{|\Lambda|}\left(\frac{e}{c}\right)^{N} \frac{\partial^{N} f_{\Lambda}}{\partial \omega^{N}}\left(\beta, \rho_{0}, \omega_{0}, \epsilon\right), \quad N \in \mathbb{N}^{*}
$$

By Corollary 3.9, $m_{\Lambda}^{N}\left(\beta, \rho_{0}, \omega_{0}, \epsilon\right)$ are well defined. Moreover:

Corollary 3.10. Let $\beta>0, \rho_{0}>0$ and $N \geq 1$. Then there exists a complex neighborhood $\mathcal{M}$ of the real axis such that $\mathcal{M} \ni \omega \mapsto m_{\Lambda}^{N}\left(\beta, \rho_{0}, \omega, \epsilon\right)$ is analytic.

Acknowledgments. This paper is dedicated to the memory of our colleague and friend Pierre Duclos (1948-2010). Part of this work has been done while B.S. was visiting Aalborg. H.C. acknowledges partial support from the Danish F.N.U. grant Mathematical Physics.

\section{References}

[1] N. Angelescu, G. Nenciu, M. Bundaru, On the Landau diamagnetism. Comm. Math. Phys. 42 (1975), 9-28.

[2] N. Angelescu, G. Nenciu, M. Bundaru, On the perturbation of Gibbs semigroups. Comm. Math. Phys. 42 (1975), 29-30.

[3] N. Angelescu, A. Corciovei, On free quantum gases in a homogeneous magnetic field. Rev. Roum. Phys. 20 (1975), 661-671

[4] J. Avron, I. Herbst, B. Simon, Schrödinger operators with magnetic fields. I. General interactions. Duke Math. J. 235 (1978), 847-883.

[5] K. Broderix, D. Hundertmark, H. Leschke, Continuity properties of Schrödinger Semigroup with magnetic fields. Rev. Math. Phys. 12 (2000), no. 2, 181-225.

[6] K. Broderix, H. Leschke, P. Müller, Continuous integral kernels for unbounded Schrödinger semigroups and their spectral projections. J. Funct. Anal. 212 (2004), no. 2, 287-323.

[7] Ph. Briet, H.D. Cornean, D. Louis, Diamagnetic expansions for perfect quantum gases. J. Math. Phys. 47 (2006), no. 8, 083511, 25 pp.

[8] Ph. Briet, H.D. Cornean, D. Louis, Generalized susceptibilities for a perfect quantum gas. Markov Process. Related Fields, 11 (2005), 177-188.

[9] Ph. Briet, H.D. Cornean, D. Louis, Diamagnetic expansions for perfect quantum gases II: uniform bounds. Asymptotic Analysis 59(1-2) (2008), 109-123. 
[10] Ph. Briet, H.D. CoRnean, Locating the spectrum for magnetic Schrödinger and Dirac operators. Comm. Partial Differential Equations 27 (2002), no. 5-6, 1079-1101.

[11] Ph. Briet, H.D. Cornean, V. Zagrebnov, Do bosons condense in a homogeneous magnetic field?, J. Statist. Phys. 116 (2004), no. 5-6, 1545-1578.

[12] M. Combescure, D. RoBert, Rigorous semiclassical results for the magnetic response of an electron gas. Rev. Math. Phys. 13 (2001), 1055-1073

[13] H.D. Cornean On the magnetization of a charged Bose gas in the canonical ensemble. Commun. Math. Phys. 212 (2000), 1-27

[14] H.D. Cornean, G. Nenciu, On the eigenfunction decay for two dimensional magnetic Schrödinger operators. Commun. Math. Phys. 192 (1998), 671-685

[15] H.D. Cornean, G. Nenciu, The Faraday effect revisited : Thermodynamic limit. J. Funct. Anal. 257 (2009), no. 7, 2024-2066

[16] H.L. Cycon, R.G. Froese, W. Kirsch, B. Simon, Schrödinger operators, with Application to Quantum Mechanics and Global Geometry. New York : Springer 1987

[17] N. Dunford And J.T. Schwartz, Linear Operators, Part II : Spectral Theory, Self Adjoint Operators in Hilbert Space. Pure and Applied Mathematics, Interscience Publishers, New York 1963

[18] S. Doi, A. Iwatsuka,T. Mine, The uniqueness of the integrated density of states for the Schrödinger operatorswith magnetic field. Math. Z., 237 (2001), 335-371.

[19] D. Gilbarg, N. S. Trudinger, Elliptic Partial Differential Equations of Second Order. Classics in Mathematics, Springer-Verlag New York, Inc., New York 1998

[20] L. Hörmander, An Introduction to Complex Analysis in Several Variables, 3rd edition. North-Holland Mathematical Library 7, North-Holland 1990

[21] B. Helffer , J. Sjöstrand, On diamagnetism and de Haas-van-Alphen effect. Ann. I.H.P. Phys. Theor. 52 (1990), 303-352

[22] K. Huang, Statistical Mechanics. Edition J. Wiley, 1987

[23] D. Hundertmark, B. Simon, A diamagnetic inequality for semigroup differences. J. Reine Angew. Math. 571 (2004), 107-130

[24] V. IftimiE, Uniqueness ans existence of the IDS for Schrödinger operators with magnetic field and elecric potential with singular negative part. Publ. Res. Inst. Math. Sci. 41 (2005), no. 2, 307-327

[25] A.M. Hinz, G. Stolz, Polynomial boundedness of eigensolutions and the spectrum of Schrödinger operators. Math. Ann. 294 (1992), 195-211.

[26] T. Kato, Perturbation Theory for Linear Operators. New York : Springer 1966

[27] M. Reed, B. Simon B, Methods of Modern Mathematical Physics II : Fourier Analysis, Self-adjointness. New York : Academic Press 1975

[28] M. Reed, B. Simon, Methods of Modern Mathematical Physics IV : Analysis of Operators. New York : Academic Press 1978

[29] B. Savoie, Ph.D thesis, in preparation.

[30] B. Simon, Schrödinger Semigroup, Bull. Amer. Math. Soc. 7 (1982), 447-526

[31] V. Zagrebnov, Topics in the Theory of Gibbs Semigroup. Leuven Notes in Mathematical and Theoretical Physics. Leuven University Press, 2003. 